\title{
The role of interactions
}

\author{
Henrique R. Schmitt ${ }^{1}$
}

${ }^{1}$ National Radio Astronomy Observatory, 520 Edgemont Road, Charlottesville, VA22903, USA email:hschmitt@nrao.edu

\begin{abstract}
Interactions between galaxies are suggested to be a mechanism responsible for feeding Active Galactic Nuclei (AGN). Theoretical models show that interactions are an efficient way to drive gas from the galaxy to the nucleus, however, the observational evidence on this subject is controversial. Here we review results in this field, discuss possible limitations of previous studies and the importance of dealing with selection effects. We also show that there is no significant difference in the percentage of low luminosity AGN and normal galaxies with companions, and discuss possible interpretations of this result.
\end{abstract}

\section{Introduction}

One of the major problems in the study of AGNs is understanding the mechanisms responsible for making the gas lose large amounts of angular momentum, move from the host galaxy toward the nucleus, and feed the black hole. Among the mechanisms that have been proposed, the principal ones are interactions, bars and nuclear spirals. The importance of bars and nuclear spirals have been addressed in several contributions to this conference (Combes, Crenshaw, Gadotti, Emsellem, Martini, Maciejewski, among others), and here we will discuss the role of interaction.

Starting with Gunn (1979), who suggested that interactions can play an important role in feeding AGNs, this mechanism has been studied by several groups, both from the theoretical and observational point of view. From the theoretical point of view, Nbody simulations by Noguchi (1987), Barnes \& Hernquist (1992), Byrd et al. (1986), Lin, Pringle \& Rees (1988), Hernquist \& Mihos (1995), Mihos \& Hernquist (1994), Taniguchi \& Wada (1996), among others, have shown that mergers, minor mergers and flybys can be responsible for bringing gas from the disk to the nuclear region. During this process the gas is shocked and compressed, leading to a period of enhanced star formation before it can be accreted by the black hole. This prediction is confirmed by the observation of higher levels of $\mathrm{H} \alpha$, infrared and radio emission in interacting galaxies (Keel et al. 1985; Kennicutt et al. 1987). Further evidence comes from the fact that luminous and ultraluminous infrared galaxies are closely related to interacting systems (Sanders \& Mirabel 1996).

On the other hand, the observational evidence for the influence of interactions in feeding AGNs is not very clear. In the case of high luminosity sources, like Quasars, there is evidence that they are related to interacting systems (Hutchings 1982; Heckman et al. 1983; Canalizo \& Stockton 2001). However, in the case of lower luminosity sources, like Seyfert galaxies, the situation is not so clear. There is no consensus on the importance of interactions in feeding their nuclei, and whether these galaxies have an excess of companions. The current results can be divided into 3 groups: those with an excess of Seyfert galaxies with companions relative to normal galaxies (e.g. Simkin, Su \& Schwarz 1980; Dahari 1984; Rafanelli, Violato \& Baruffolo 1995; Laurikainen et al. 1994); those with no difference between Seyferts and normal galaxies (Fuentes-Williams \& Stocke 1988; Ulvestad \& Wilson 1984; De Robertis, Yee \& Hayhoe 1998); and those with a 
higher percentage of Seyfert 2s than Seyfert 1s with companions (Laurikainen \& Salo 1995, and Dultzin-Hacyan et al. 1999).

We believe that the contradictory results obtained by different papers are most likely due to the way they selected their samples and control samples, as pointed out by Heckman (1990). A major requirement for one to be able to do a proper comparison between the percentage of AGN and normal galaxies with companions, is to have a sample of active and control galaxies matched by their host properties. Previous studies also had to deal with the fact that they usually did not have information about the redshifts of nearby galaxies projected on the sky, so they were not always able to know if these galaxies were physically associated. This required the application of statistical corrections for the number of background and foreground objects projected around the galaxies being studied.

\section{Low Luminosity AGN}

In this section we present the results of a recent study of the percentage of companions in galaxies with different activity types (Schmitt 2001), where most of the selection effects, as well as other problems pointed out above, were avoided. Instead of using the more traditional technique of selecting a control sample of galaxies to match the AGN one, the approach used in this paper was to employ a sample of galaxies selected by their host properties, independent of the fact of being active or not. The sample selected for this study was the Palomar survey (Ho, Filippenko \& Sargent 1997a), which comprises all the galaxies brighter than $\mathrm{B}_{T}=12.5 \mathrm{mag}$ in the northern hemisphere. This sample is ideal for this work because it includes both the AGN and the control sample in itself.

Besides mitigating the sample selection problem, the Palomar sample presents several additional advantages relative to other samples. It provides homogeneous high-quality spectroscopic measurements of emission line fluxes, and activity classification of all the galaxies. This sample contains a large enough number of galaxies, with a large range of parameters (e.g. host galaxy morphology and nuclear luminosity) ensuring a significant comparison between the different activity types, and allowing us to draw robust statistical conclusions. For a detailed discussion of other advantages of this sample relative to other ones commonly used in the literature, and for a discussion of possible selection biases which affect them, see Ho \& Ulvestad (2001).

Starting with the original sample of 486 galaxies from Ho et al. (1997a), we excluded local group galaxies and all those with $\mathrm{B}_{T}>12.5 \mathrm{mag}$, thus reducing the total number of objects to 451 . These galaxies were divided in 5 groups, according to their nuclear activity type. The groups and the number of galaxies in each one of them are: 46 Seyferts, 193 HII galaxies, 88 LINERs, 63 Transition objects and 61 Absorption line galaxies. We point out that most of the HII galaxies in this sample have only quiescent star formation. Also, we do not try to separate the Seyfert galaxies into Seyfert 1s and Seyfert 2s.

The influence of interactions on AGN activity was studied using two techniques. First we compared the environments where galaxies with different activity types are found. This was done using the local galaxy density parameter $\left(\rho_{\text {gal }}\right)$, which was defined by Tully (1988) as the density of galaxies brighter than $\mathrm{M}_{B}=-16 \mathrm{mag}$ in the vicinity of the object of interest. We found that the different activity types have similar distributions of $\rho_{\text {gal }}$ values, indicating that there is no significant difference in their environments (see the contribution by Maia for results on a different sample).

The second technique consisted of searching for companions around the galaxies in the Palomar sample. This was done using NED and the Digitized Sky Survey images. The criteria used to determine if a galaxy has a companion are similar to the ones used by 


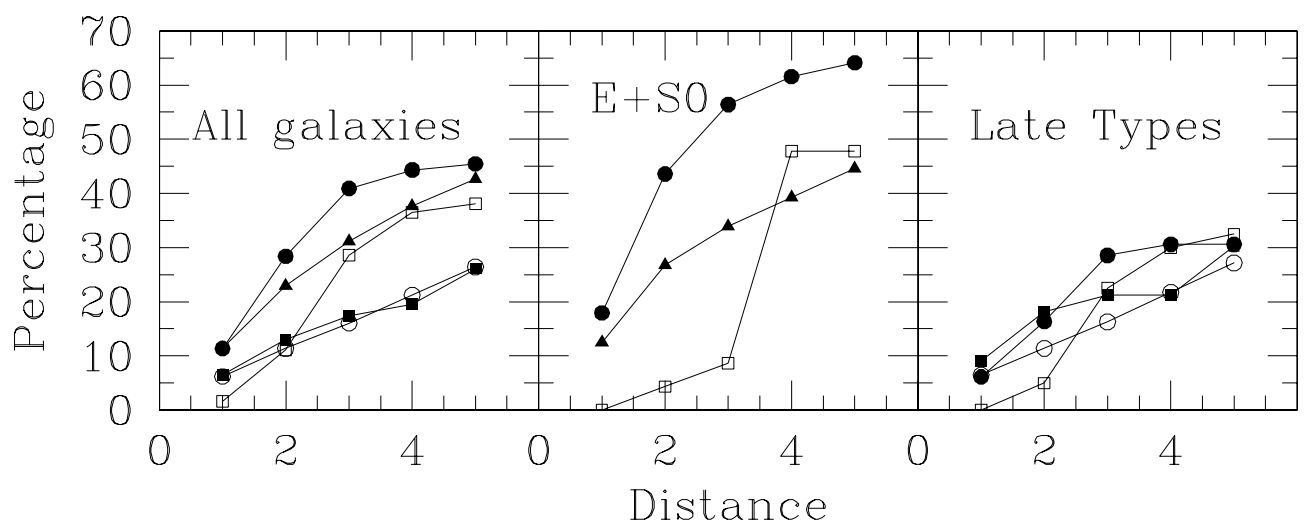

Figure 1. Percentage of galaxies with companions as a function of the distance between the two galaxies, in units of the diameter of the primary galaxy. LINER's are represented by filled circles, Transition galaxies by open squares, Absorption line galaxies by filled triangles, Seyfert galaxies by filled squares and HII galaxies by open circles. The left panel shows the results for all the galaxies, the middle panel shows the results for Elliptical and S0 galaxies only, while the right panel shows the results for late type galaxies only.

Rafanelli et al. (1995), but modified to allow a slightly larger search region. We determine that a galaxy is a companion if its distance to the galaxy of interest, the primary galaxy, is smaller than 5 times the diameter $\left(D_{25}\right)$ of that galaxy, the difference in brightness between them is smaller than 3 magnitudes $(|\Delta m| \leqslant 3 \mathrm{mag})$, and the difference in radial velocities is smaller than $1000 \mathrm{~km} \mathrm{~s}^{-1}\left(\left|\Delta V_{R a d}\right| \leqslant 1000 \mathrm{~km} \mathrm{~s}^{-1}\right)$. The Palomar sample presents a major advantage, relative to most samples, in the latter criterion. Since redshift surveys such as the CfA2 (Falco et al. 1999) are observing galaxies up to $B \leqslant 15.5$, we are able to obtain radial velocity information even for the faintest possible companions of galaxies in our sample.

Considering that most of the galaxies in this sample have $\mathrm{M}_{B}<-19$, we can calculate that this technique is sensitive to companions as faint as $\mathrm{M}_{B} \sim-16$, which is similar to the absolute magnitude of the $\operatorname{SMC}\left(\mathrm{M}_{B}=-16.3\right)$. For more typical galaxies, with magnitudes around $\mathrm{M}^{*}\left(\mathrm{M}_{B}^{*}=-20.3\right)$, this technique is sensitive to companions slightly fainter than the LMC $\left(\mathrm{M}_{B}=-18.5\right)$. However, we are not sensitive to lower luminosity dwarf spheroidal and irregular galaxies, which were suggested by De Robertis et al. (1998) as possible triggers of activity in Seyfert galaxies.

The results from the comparison of the percentage of galaxies with companions among the different activity types are presented in Figure 1. We find in the left panel of this figure that, when all the galaxies in the sample are considered, there is a similar percentage of Seyfert and HII galaxies with companions. However, a surprising result arises when we compare these percentages with those for LINER's, Transition and Absorption galaxies, which present significantly larger percentages of companions, by a factor of approximately two. A detailed statistical comparison between the different activity types is presented in Schmitt (2001).

This result seems to contradict all previous results available in the literature, which indicated that the percentage of Seyfert and HII galaxies with companions was either similar to or higher than that of other galaxies. However, this contradiction is solved when we take into account the morphological types of the galaxies in our sample. Ho et al. (1997b) showed that different activity types have different distributions of morphological types. A particularly important result of their analysis is the fact that most Absorption 
galaxies and a significant percentage of LINERs and Transition galaxies are found in Elliptical or S0 hosts. On the other hand, almost all HII galaxies and a high percentage of Seyferts have morphological types Sa or later. Since the percentage of elliptical galaxies increases with the density of the environment, what is known as the morphology-density effect (Dressler 1980, Charlton et al. 1995; Budavári et al. 2003), one would expect to find a higher percentage of elliptical than spiral galaxies with companions. As a consequence of this effect, the percentage of LINER, Transition and Absorption galaxies with companions should on average be higher than in the case of Seyfert and HII galaxies.

To address this issue we separate our sample into two groups, those with morphological types E and S0, and those with morphological types Sa and later. The results are presented in the middle and right panels of Figure 1, respectively. The middle panel shows the percentage of early type galaxies with companions, as a function of the separation between the galaxies, where we can see that LINER, Transition and Absorption line galaxies have similar percentages of companions. This panel does not include Seyfert and HII galaxies because the sample does not have many of these galaxies in Ellipticals and S0's hosts. The comparison between late type galaxies shows that all activity classes have similar percentages of galaxies with companions. Similar results were found by Ho, Filippenko \& Sargent (2003), using only galaxies with morphological types between Sab and Sbc. Comparing the percentage of early and late type LINER's with companions we find a frequency 2 times higher in the former, indicating that the contradictory results obtained using all galaxies in the sample were due to the mismatch of morphological types of the different activity classes.

Finally, we address whether there is a difference in the percentage of Seyfert 1s and Seyfert 2s with companions. Laurikainen \& Salo (1995) and Dultzin-Hacyan et al. (1999) found that, when compared to normal galaxies, Seyfert 1s have a similar percentage of companions, while for Seyfert 2s this percentage doubles. However, as discussed in Schmitt et al. (2001), their results can be attributed to the way their samples were selected, based on ultraviolet excess. Since the nucleus of Seyfert $2 \mathrm{~s}$ is hidden from direct view, the amount of ultraviolet emission reflected in our direction is very small, indicating that the ultraviolet excess detected in their Seyfert $2 \mathrm{~s}$ is due to a source outside the torus, like a starburst. Since strong star formation is related to interactions (see next section), and a large percentage $(\sim 40 \%)$ of Seyfert galaxies are known to have circumnuclear star formation (Cid Fernandes et al. 2001), it is likely that their results were influenced by selection effects. In fact, Schmitt et al. (2001) show that, when we use a sample of Seyfert galaxies selected from an isotropic property, $60 \mu \mathrm{m}$ luminosity, both Seyfert types have similar percentages of galaxies with companions. More recently, Ho et al. (2003) found a higher percentage of Seyfert 1s than Seyfert 2s with companions in the Palomar sample. Nevertheless, they note that this result can be due to the fact that some broad-lined Seyfert galaxies in their sample are actually Seyfert $2 \mathrm{~s}$. Due to the high sensitivity of this survey, they were able to detect the reflected broad lines in some Seyfert 2 galaxies like in NGC1068, in direct light. Taking into account cases like this might in fact remove the difference between the two Seyfert types.

\section{Higher Luminosities and Distances}

The results presented in the previous section indicate that there is no significant difference in the percentage of normal galaxies and low luminosity AGNs with companions, or in their environments. However, one might argue that interactions may be more important for higher luminosity AGNs, or for sources at higher redshifts. In the case of HII galaxies, we find that when we take into account only the objects with $\mathrm{L}(\mathrm{H} \alpha)>10^{40} \mathrm{erg}$ 
$\mathrm{s}^{-1}$ in the Palomar sample, $65 \%$ of them have companions. For comparison, this percentage drops to $\sim 30 \%$ for lower luminosity sources. This result is consistent with those from Kennicutt et al. (1987) and Keel et al. (1985), indicating that interactions can be responsible for the enhancement of the luminosity of these galaxies.

In the case of AGNs, studies by Brown et al. (2001), Miller et al. (2003) and Grogin et al. (2003), based on strong AGNs at higher redshifts, found that there is no difference in the environment or the number of companions around these galaxies compared to normal ones. Nevertheless, a different result was obtained by Kauffmann et al. (2004), based on a much larger sample of AGNs extracted from the Sloan Survey. They found a higher fraction of luminous AGN in lower density environments, which indicates that the fraction of galaxies able to host strong AGN decreases in high-density environments.

\section{Discussion and Future Work}

We saw in the previous sections that there is no difference in the percentage of AGNs and normal galaxies with companions. Combining this fact with the lack of an excess of AGNs, relative to normal galaxies, with bars or nuclear spirals, one can interpret these results as evidence for these mechanisms not being important in the AGN feeding process. However, a different interpretation, in particular for the case of interactions, have been proposed by several authors (Corbin 2000; Schmitt 2001; Storchi-Bergmann et al. 2001; see also the contributions by Martini, and Storchi-Bergmann et al.). These authors suggest that these mechanisms play an important role in the feeding process, but there is a delay between the time when the mechanism is active and when the gas is being accreted by the nuclear black hole, indicating that the nuclear activity may be cyclic and the AGNs evolve with time.

Support for this scenario comes from several sources. The results obtained from N-body simulations (e.g. Byrd et al. 1986; Hernquist \& Mihos 1995) indicate that an interaction first moves gas toward the nucleus, where its temperature and density increases and a period of strong star formation happens. Later on this process the gas loses more angular momentum and is accreted by the black hole, with the galaxy being detect as an AGN. Taking into account the fact that all galaxies with bulges are believed to have massive black holes in their nuclei (Magorrian et al. 1998, Gebhardt et al. 2000, Ferrarese \& Merritt 2000) it is reasonable to assume that when an interaction happens they can pass through different periods of activity. First one would detect an HII like nucleus, which could later evolve into a period of Seyfert activity, when the gas is being accreted by the black hole at a high rate, and finally become a LINER or transition galaxy, when the amount of gas available to feed the nucleus is small. Observational evidence in favor of this scenario comes from the detection of recent star formation, with ages around $100 \mathrm{Myr}$, in the nuclear region of at least $40 \%$ of Seyfert and LINER/HII galaxies (Cid Fernandes et al. 2001, and this conference).

The importance of interactions for the proposed scenario can be tested observationaly. A relatively easy test is to compare the asymmetry indices (Conselice 1997) of Seyfert and normal galaxies. If interactions play an important role in feeding AGNs, one would expect larger asymmetries in Seyfert galaxies compared to normal ones, or an evolution of this index from one activity type to another. Similarly, it is also possible to use HI $21 \mathrm{~cm}$ to detect the signature of interactions no longer detectable in optical images. The contribution by Lim et al. shows the results of such a study, where they find that Seyfert galaxies present much more distorted and extended HI emission than normal galaxies. It would be important to extend this study to a larger, well defined sample. 


\section{Acknowledgements}

The National Radio Astronomy Observatory is a facility of the National Science Foundation, operated under cooperative agreement by Associated Universities, Inc.

\section{References}

Barnes, J. E., \& Hernquist, L. 1992, ARA\&A, 30, 705

Brown, M. J. I., Boyle, B. J., \& Webster, R. L. 2001, AJ, 122, 26

Budavári, T., et al. 2003, ApJ, 595, 59

Byrd, G. G., Valtonen, M. J., Valtaoja, L., \& Sundelius, B. 1986, A\&A, 166, 75

Canalizo, G., \& Stockton, A. 2001, ApJ, 555, 719

Cid Fernandes, R., Heckman, T., Schmitt, H., Delgado, R. M. G., \& Storchi-Bergmann, T. 2001, ApJ, 558, 81

Charlton, J. C., Whitmore, B. C., \& Gilmore, D. M. 1995, in Groups of Galaxies, ASP Conf. Ser., 70,49

Conselice, C. J. 1997, PASP, 109, 1251

Corbin, M. R. 2000, ApJ, 536, L73

Dahari, O. 1984, AJ, 89, 966

de Robertis, M. M., Yee, H. K. C., \& Hayhoe, K. 1998, ApJ, 496, 93

Dressler, A. 1980, ApJ, 236, 351

Dultzin-Hacyan, D., et al. 1999, ApJ, 513, L111

Falco, E. E., et al. 1999, PASP, 111, 438

Ferrarese, L., \& Merritt, D. 2000, ApJ, 539, L9

Fuentes-Williams, T., \& Stocke, J. T. 1988, AJ, 96, 1235

Gebhardt, K., et al. 2000, ApJ, 539, L13

Grogin, N. A., et al. 2003, ApJ, 595, 685

Gunn, J. E. 1979, Active galactic nuclei, Cambridge, Cambridge University Press, 213

Heckman, T. M. 1990, IAU Colloq. 124: Paired and Interacting Galaxies, p.359

Heckman, T. M., Bothun, G. D., Balick, B., \& Smith, E. P. 1984, AJ, 89, 958

Hernquist, L., \& Mihos, J. C. 1995, ApJ, 448, 41

Ho, L. C., Filippenko, A. V., \& Sargent, W. L. W. 1997a, ApJS, 112, 315

Ho, L. C., Filippenko, A. V., \& Sargent, W. L. W. 1997b, ApJ, 487, 568

Ho, L. C., Filippenko, A. V., \& Sargent, W. L. W. 2003, ApJ, 583, 159

Ho, L. C., \& Ulvestad, J. S. 2001, ApJS, 133, 77

Hutchings, J. B. 1983, PASP, 95, 799

Kauffmann, G., et al. 2004, MNRAS, in press (astro-ph/0402030)

Keel, W. C., Kennicutt, R. C., Hummel, E., \& van der Hulst, J. M. 1985, AJ, 90, 708

Kennicutt, R. C. et al. 1987, AJ, 93, 1011

Laurikainen, E., \& Salo, H. 1995, A\&A, 293, 683

Laurikainen, E., Salo, H., Teerikorpi, P., \& Petrov, G. 1994, A\&AS, 108, 491

Lin, D. N. C., Pringle, J. E., \& Rees, M. J. 1988, ApJ, 328, 103

Magorrian, J., et al. 1998, AJ, 115, 2285

Mihos, J. C., \& Hernquist, L. 1994, ApJ, 425, L13

Miller, C. J., Nichol, R. C., Gómez, P. L., Hopkins, A. M., \& Bernardi, M. 2003, ApJ, 597, 142

Noguchi, M. 1987, MNRAS, 228, 635

Rafanelli, P., Violato, M., \& Baruffolo, A. 1995, AJ, 109, 1546

Sanders, D. B., \& Mirabel, I. F. 1996, ARA\&A, 34, 749

Schmitt, H. R. 2001, AJ, 122, 2243

Schmitt, H. R. et al. 2001 ApJ, 555, 663

Simkin, S. M., Su, H. J., \& Schwarz, M. P. 1980, ApJ, 237, 404

Storchi-Bergmann, T., et al. 2001, ApJ, 559, 147

Taniguchi, Y., \& Wada, K. 1996, ApJ, 469, 581

Tully, R. B. 1988, in Nerby Galaxy Catalog, Cambridge, Cambridge University Press

Ulvestad, J. S., \& Wilson, A. S. 1984, ApJ, 285, 439 